\title{
INVERSOR ATS
}

\author{
Euzeli Cipriano dos Santos Júnior \\ Universidade Federal de Campina Grande \\ Departamento de Engenharia Elétrica \\ Campina Grande, PB - Brasil \\ e-mail: euzeli@dee.ufcg.edu.br
}

\begin{abstract}
Resumo - Neste artigo é apresentada uma nova topologia de inversor baseado na ação de tensão série (ATS) usado para gerar níveis de tensão adicionais. Este inversor será chamado de Inversor ATS. O Inversor ATS apresenta uma série de vantagens sobre os inversores tradicionais, tais como tensão de saída maior que a tensão no barramento cc e menor distorção harmônica na tensão de saída. $\mathrm{O}$ circuito proposto emprega dois inversores com três braços de dois níveis conectados num arranjo série através dos transformadores de alta freqüência. Na análise apresentada neste artigo inclui-se o modelo do inversor e sua operação, além da estratégia PWM (Pulse Width Modulation). Uma comparação geral com inversores convencionais é também apresentada. Resultados de simulação e experimentais corroboram com o estudo teórico.
\end{abstract}

Palavras-Chave - Conversor cc-ca, Inversor Fonte de Tensão, Configuração de Inversor.

\section{SVA INVERTER}

Abstract - In this paper is presented a new inverter topology based on series-voltage-action (SVA) used to generate additional levels. This inverter will be called as SVA Inverter. The SVA Inverter presents some advantages over the traditional inverters, such as output voltage higher than the dc-link voltage and lower distortion in output voltage. The proposed circuit employs two three-leg two-level inverters connected in a series arrangement through high-frequency transformers. The complete analysis presented in this paper includes the inverter model and its operation and PWM (Pulse Width Modulation) strategy. A general comparison with conventional inverter is also presented. Simulated and experimental results corroborate the theoretical study.

Keywords - Dc-ac Converter, Voltage Source Converter, Inverter Configuration.

Artigo submetido em 25/09/2010. Primeira revisão em 13/05/2011; segunda revisão em 26/07/2011. Aceito para publicação em 15/08/2011 por recomendação do editor João Onofre P. Pinto.

\section{INTRODUÇÃO}

Topologias de conversores cc-ca podem ser consideradas como uma tecnologia estabelecida com soluções para diferentes aplicações. Por exemplo, em aplicações no qual é exigido uma tensão de saída com baixa distorção harmônica, as configurações multi-níveis podem ser usadas com diferentes circuitos [1]: diodo grampeado [2-4],[28], capacitor flutuante [5-7], e conexão de conversores em cascata com fontes cc separadas [8-16] ou com uma única fonte cc [17]. Outra possibilidade para reduzir a distorção da corrente de saída é usar a técnica de interleaved para conversores conectados em paralelo [18], [19], [27]. Como aplicação dos conversores cc-ca podemos citar: sistemas de acionamento de motor linear [29], processador de energia em sistemas com energias renováveis [30,31] e sistemas tolerantes a falhas [32].

Recentemente o custo das chaves de potência tem diminuído substancialmente [20], o que permite o uso de topologias com elevado número de chaves sem aumentar dramaticamente o preço final do conversor. Além disto, o valor inicial alto pode ser justificado se a THD (Total Harmonic Distortion) da tensão de saída é considerado um aspecto importante na escolha do inversor.

Este artigo apresenta uma nova topologia de inversor baseado na ação de tensão série (ATS) para ser usado em aplicações de baixa potência, mas que exija baixos níveis de THD da tensão aplicada à carga, como por exemplo, em sistemas de acionamento de alta velocidade, onde a baixa THD das variáveis geradas pelo conversor é de fundamental importância, já que a frequêencia da modulante é da mesma ordem de grandeza da frequiência da portadora. Outra possível aplicação seria em sistemas fotovoltaicos, onde normalmente existe a demanda por conversores que processem uma tensão de saída maior que aquela obtida na entrada, ou mesmo a utilização do conversor proposto como retificador controlado, de forma que a carga seria substituída pela fonte trifásica e a bateria seria substituída por uma carga do tipo cc.

O Inversor ATS é constituído de doze chaves de potência e três transformadores de alta freqüência monofásicos, como observado na Figura 1. O circuito proposto emprega dois inversores de três braços de dois níveis conectados num arranjo série através dos transformadores. Apesar de usar um número relativamente baixo de chaves de potência, o Inversor ATS produz uma tensão de fase na carga com sete níveis, o que melhora substancialmente a THD da tensão de saída. Outra vantagem do Inversor ATS é a possibilidade de 


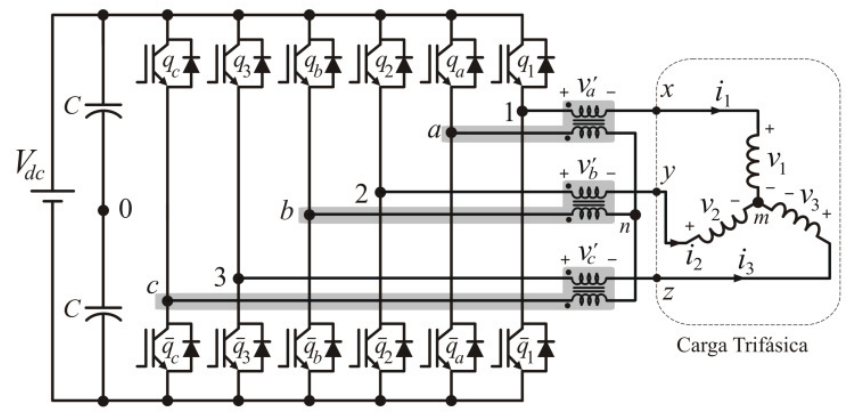

Fig. 1. Inversor ATS.

gerar tensões de saída maiores que a tensão do barramento cc.

\section{OPERAÇÃO DO INVERSOR ATS}

O circuito equivalente do Inversor ATS é apresentado na Figura 2(a). Na Figura 2(b) mostra-se a fase 1 do circuito proposto destacando as tensões associadas com esta fase. A relação de transformação dos transformadores mostrados na Figura 1 é de 1:1, esta relação de transformação garante uma relação ótima entre THD da tensão aplicada a carga e baixas perdas do conversor. As tensões de pólo vista pela carga trifásica $\left(v_{x 0}, v_{y 0}\right.$ e $\left.v_{z 0}\right)$ podem ser escritas por

$$
\begin{aligned}
& v_{x 0}=v_{10}-v_{a}^{\prime}=v_{10}-v_{a} \\
& v_{y 0}=v_{20}-v_{b}^{\prime}=v_{20}-v_{b} \\
& v_{z 0}=v_{30}-v_{c}^{\prime}=v_{30}-v_{c}
\end{aligned}
$$

onde as tensões $v_{10}, v_{20}$ e $v_{30}$ são as tensões de pólo associadas com os braços 1, 2 e 3 respectivamente. Sabe-se que as tensões de pólo do circuito da Figura 1 podem assumir apenas dois valores $\pm V_{d c} / 2$, como observado na Figura 2 (c). Por outro lado, as tensões de fase aplicadas no primário dos transformadores, ou seja, $v_{a n}, v_{b n}$ e $v_{c n}$ podem assumir cinco diferentes valores (ou níveis): $\pm 2 V_{d c} / 3, \pm V_{d c} / 3$ e 0 , como observado na Figura 2(d).

Conseqüentemente, as tensões de pólo vista pela carga trifásica $\left(v_{x 0}, v_{y 0}\right.$ e $\left.v_{z 0}\right)$ têm oito níveis, como será mostrado a seguir.

As tensões aplicadas no primário dos transformadores são dadas por

$$
\begin{aligned}
& v_{a}=v_{a 0}-v_{n 0} \\
& v_{b}=v_{b 0}-v_{n 0} \\
& v_{c}=v_{c 0}-v_{n 0}
\end{aligned}
$$

onde $v_{a 0}, v_{b 0}$ e $v_{c 0}$ são as tensões de pólo associadas com os braços $a, b$ e $c$, respectivamente. Somando-se (4)-(6) e admitindo tensões trifásicas equilibradas $v_{a}+v_{b}+v_{c}=0$, obtém-se $v_{n 0}=\frac{1}{3}\left(v_{a 0}+v_{b 0}+v_{c 0}\right)$. Então

$$
\begin{aligned}
& v_{a}=\frac{2 v_{a 0}}{3}-\frac{v_{b 0}}{3}-\frac{v_{c 0}}{3} \\
& v_{b}=-\frac{v_{a 0}}{3}+\frac{2 v_{b 0}}{3}-\frac{v_{c 0}}{3} \\
& v_{c}=-\frac{v_{a 0}}{3}-\frac{v_{b 0}}{3}+\frac{2 v_{c 0}}{3}
\end{aligned}
$$

Substituindo (7)-(9) em (1)-(3), as tensões de pólo vista pela carga serão dadas por

$$
\begin{aligned}
& v_{x 0}=v_{10}-\frac{2 v_{a 0}}{3}+\frac{v_{b 0}}{3}+\frac{v_{c 0}}{3} \\
& v_{y 0}=v_{20}+\frac{v_{a 0}}{3}-\frac{2 v_{b 0}}{3}+\frac{v_{c 0}}{3} \\
& v_{z 0}=v_{30}+\frac{v_{a 0}}{3}+\frac{v_{b 0}}{3}-\frac{2 v_{c 0}}{3}
\end{aligned}
$$

As equações (10)-(12) mostram que as tensões de pólo $\left(v_{x 0}\right.$, $v_{y 0}$ e $\left.v_{z 0}\right)$ podem assumir oito diferentes níveis $\left( \pm 7 V_{d c} /\right.$ $\left.6, \pm 5 V_{d c} / 6, \pm 3 V_{d c} / 6, \pm V_{d c} / 6\right)$.

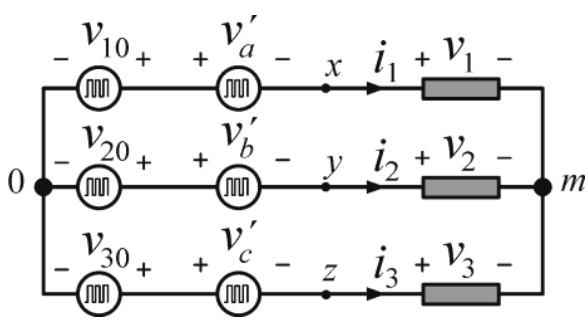

(a)

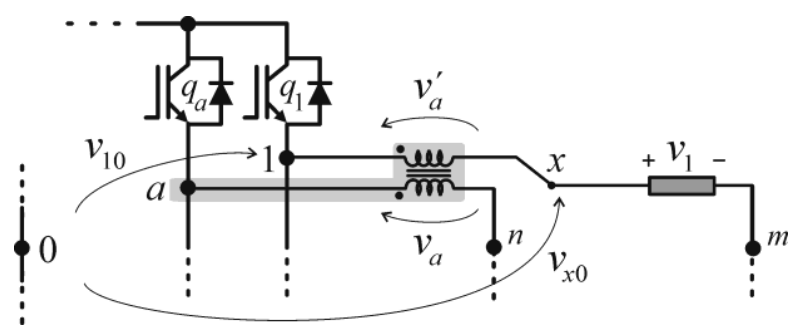

(b)

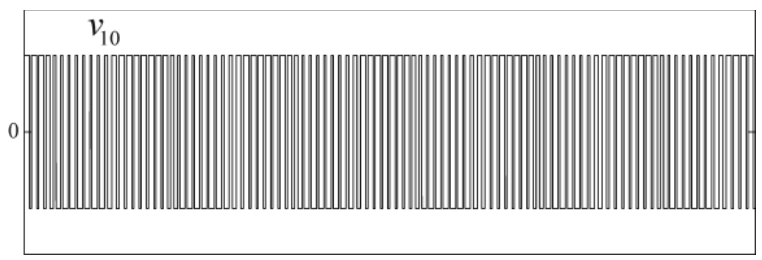

(c)

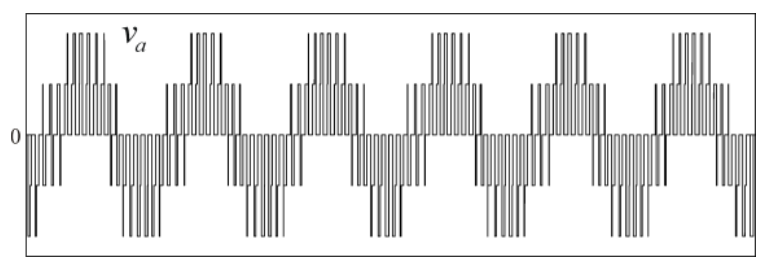

(d)

Fig. 2. (a) Circuito equivalente. (b) Fase 1 do circuito destacando as tensões associadas com esta fase. (c) Tensão de dois níveis $v_{10}$. (d) Tensão de cinco níveis $v_{a}$. 


\section{ESTRATÉGIA PWM}

A geração de tensão PWM do Inversor ATS pode ser implementada usando as tensões de fase de referência. Assim, considerando as tensões de fase de referência dadas por (13)-(18) com $v_{a}^{*}=-v_{1}^{*}, v_{b}^{*}=-v_{2}^{*}$ e $v_{c}^{*}=-v_{3}^{*}$.

$$
\begin{aligned}
& v_{10}^{*}=v_{1}^{*}+v_{m 0}^{*} \\
& v_{20}^{*}=v_{2}^{*}+v_{m 0}^{*} \\
& v_{30}^{*}=v_{3}^{*}+v_{m 0}^{*}
\end{aligned}
$$

e

$$
\begin{aligned}
& v_{a 0}^{*}=v_{a}^{*}+v_{n 0}^{*} \\
& v_{b 0}^{*}=v_{b}^{*}+v_{n 0}^{*} \\
& v_{c 0}^{*}=v_{c}^{*}+v_{n 0}^{*}
\end{aligned}
$$

As equações (13)-(15) e (16)-(18) não podem ser resolvidas sem que as tensões $v_{m 0}^{*}$ e $v_{n 0}^{*}$ sejam especificadas. Estas tensões $\left(v_{m 0}^{*}\right.$ e $\left.v_{n 0}^{*}\right)$ podem ser calculadas em função do fator de distribuição de roda livre $\mu$, como é feito em um inversor trifásico convencional [21], [22].

Por exemplo, a tensão $v_{m 0}^{*}$ pode ser calculada levando-se em consideração a expressão abaixo:

$$
v_{m 0}^{*}=E\left(\mu-\frac{1}{2}\right)-\mu v_{\text {max }}^{*}+(\mu-1) v_{\text {min }}^{*}
$$

onde $v_{\text {max }}^{*}=\max \{V\}, v_{\text {min }}^{*}=\min \{V\}, \operatorname{com} V$ dado por: $V=\left\{v_{1}^{*}, v_{2}^{*}, v_{3}^{*}\right\}$ e $E$ é a tensão do barramento capacitivo. A expressão (19) foi derivada usando a mesma estratégia PWM para o conversor trifásico [21], [22].

O fator de distribuição de roda livre $\mu(0 \leq \mu \leq 1)$ é dado por $\mu=t_{o i} / t_{o}$, este parâmetro divide igualmente o período de aplicação do vetor de roda livre no começo e no final do período de chaveamento. A mesma estratégia tem sido utilizada para o cálculo da tensão $v_{n 0}^{*} \operatorname{com} V=\left\{v_{a}^{*}, v_{b}^{*}, v_{c}^{*}\right\}$.

$\mathrm{O}$ diagrama de blocos para geração das tensões PWM é mostrado na Figura 3. Nesta figura $v_{\text {carrier }}$ é uma forma de onda triangular de alta freqüência.

As tensões geradas pelo conversor podem ser representadas em um diagrama vetorial. O primeiro passo para obtenção dessa representação vetorial das tensões sintetizadas pelo conversor é a partir da determinação das tensões de fase da carga $\left(v_{1}, v_{2}, v_{3}\right)$ em função das tensões de pólo vista pela carga $\left(v_{x 0}, v_{y 0}, v_{z 0}\right)$, ou seja, partir das equações (10)-(12). Como estas tensões são funções dos estados das chaves do conversor, é possível escrever as tensões $d q$ também em função dos estados das chaves. $\mathrm{Na}$ seqüência determinam-se as tensões resultantes no plano $d q$ para cada combinação de chaveamento válida, como observado na Figura 4.

\section{ESPECIFICAÇ̃̃O DO TRANSFORMADOR}

Alguns trabalhos encontrados na literatura técnica utilizam transformadores de alta freqüência na saída do inversor [25], [26], mas com arranjo de conexão diferente daquele apresentado pelo Inversor ATS. A metodologia de projeto dos transformadores monofásicos de alta freqüência, empregados no Inversor ATS, pode seguir a mesma estratégia empregada em [25], [26].

A presença destes transformadores na saída do inversor restringe sua aplicação em termos da capacidade de potência fornecida pelo inversor, já que o projeto dos transformadores de alta freqüência em alta potência poderia inviabilizar a proposta. No entanto, em sistemas de baixa potência, a topologia proposta apresenta características interessantes não observadas em outras configurações da literatura técnica.

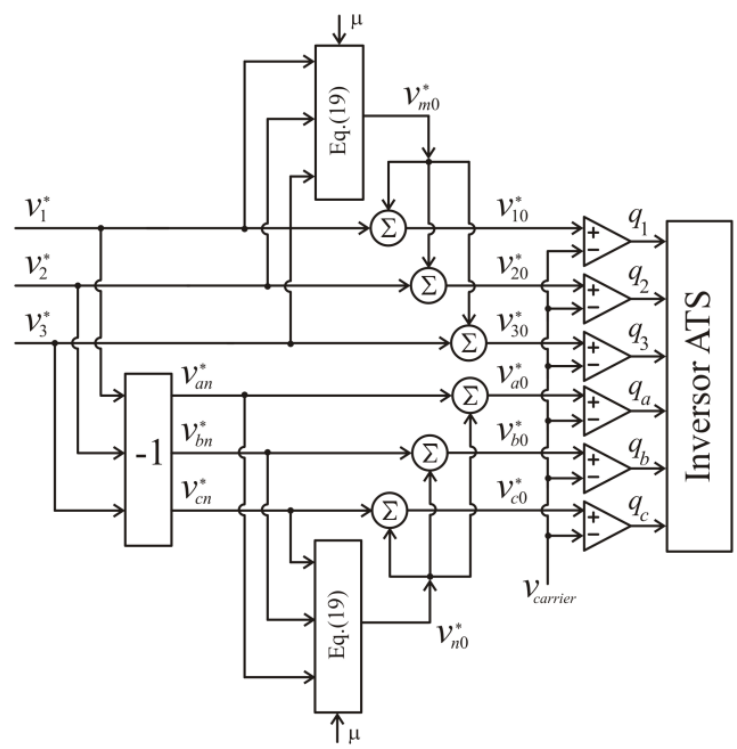

Fig. 3. Diagrama de blocos para geração dos sinais PWM.

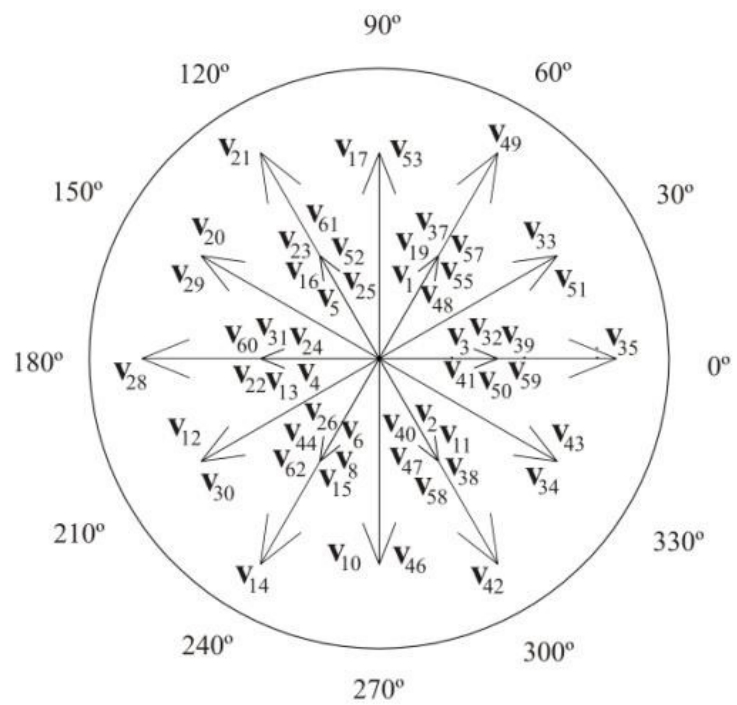

Vetores Nulos: $\mathbf{v}_{0}, \mathbf{v}_{7}, \mathbf{v}_{9}, \mathbf{v}_{18}, \mathbf{v}_{27}, \mathbf{v}_{36}, \mathbf{v}_{45}, \mathbf{v}_{54}, \mathbf{v}_{56}, \mathbf{v}_{63}$

Fig. 4. Diagrama fasorial mostrando todas as tensões disponíveis no plano complexo.

A especificação dos transformadores é determinada pelas variáveis do conversor cc-ca utilizado e pela carga, ou seja, a tensão e a corrente dos transformadores são dadas respectivamente por $V_{d c} / \sqrt{3}$ e $i_{\alpha}$ (com $\alpha=1,2,3$ - corrente na carga), enquanto a freqüência é definida pela freqüência de chaveamento do conversor. Em termos de potência, cada transformador irá processar $1 / 6$ da potência da carga. 


\section{RESULTADOS DE SIMULAÇÕES E EXPERIMENTAIS}

Mostra-se nas Figuras. 5 e 6 um conjunto de resultados simulados do Inversor ATS, com freqüência de chaveamento igual a $10 \mathrm{kHz}$ e com tensão no barramento cc de $70 \mathrm{~V}$. Na Figura 5 são apresentadas as seguintes variáveis: tensão de pólo do braço $1\left(v_{10}\right)$, tensão de fase no primário do transformador $\left(v_{a}\right)$, tensão de pólo vista pela carga $\left(v_{x 0}\right)$ e a tensão de fase da carga $\left(v_{1}\right)$. Todas estas variáveis são relacionadas com a fase 1 e a relação de transformação dos transformadores de alta freqüência é dada por 1:1. Note que a quantidade de níveis das tensões de pólo vista pela carga possui 8 níveis, como esperado no estudo teórico. Além disto, percebe-se que a tensão de saída é maior que a tensão do barramento cc.

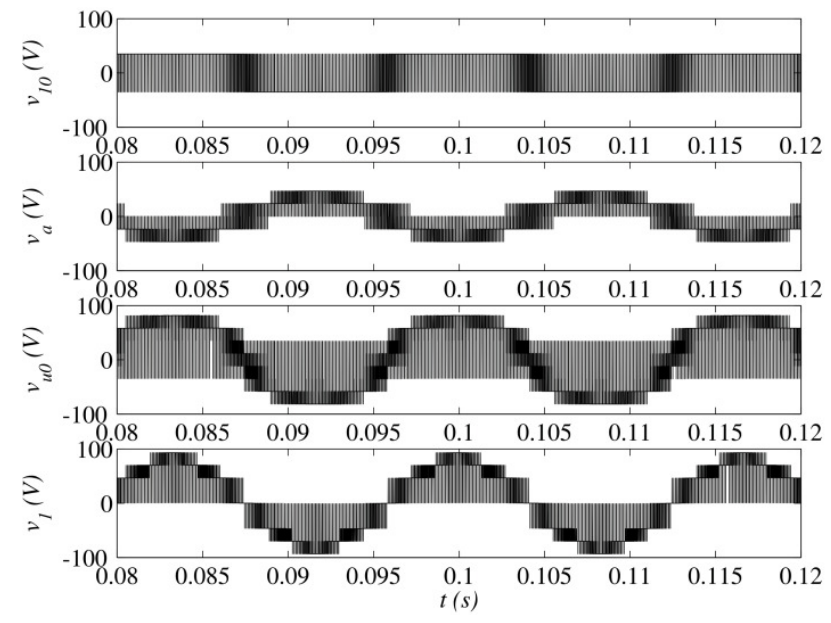

Fig. 5. Resultados de simulação: variáveis relacionadas com a fase 1 do Inversor ATS.

Apresenta-se na Figura 6 as tensões e as correntes nas fases da carga trifásica.

Mostra-se na Figura 7 os resultados experimentais das tensões e da corrente de fase da carga trifásica sendo alimentado pelo Inversor ATS. Estes resultados foram obtidos com os seguintes parâmetros: freqüência de chaveamento igual a $10 \mathrm{kHz}$, tensão cc obtida a partir de retificador trifásico a diodos e capacitor de $2200 \mu F$, carga $R L \quad(R=6.5 \Omega$ e $L=7 \mathrm{mH})$, chave de potência IGBT da SEMIKRON com SKHI-23. Note que tanto os resultados de simulação quanto os resultados experimentais são concordantes com a expectativa levantada teoricamente.

Mostra-se na Figura 8(a) a tensão de pólo $v_{10}$ e a corrente no secundário do transformador, ou seja, a corrente que circula no braço 1, enquanto na Figura 8(b), mostra-se a tensão de pólo $v_{a 0}$ e a corrente no primário do transformador, ou seja, a corrente que circula no braço $a$. $\mathrm{O}$ resultado experimental mostrado na Figura 8 ilustra que a potência processada pelo inversor ATS é igualmente distribuída entre os braços do conversor, já que o nível de corrente e o padrão das tensões de pólo são semelhantes para os dois braços.

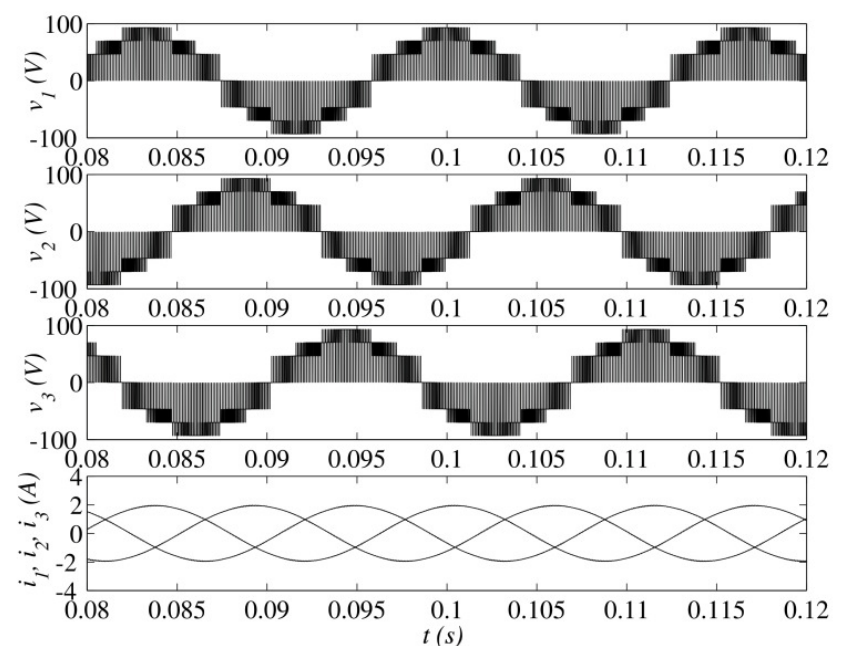

Fig. 6. Resultados de simulação: tensões e correntes aplicadas a carga trifásica.

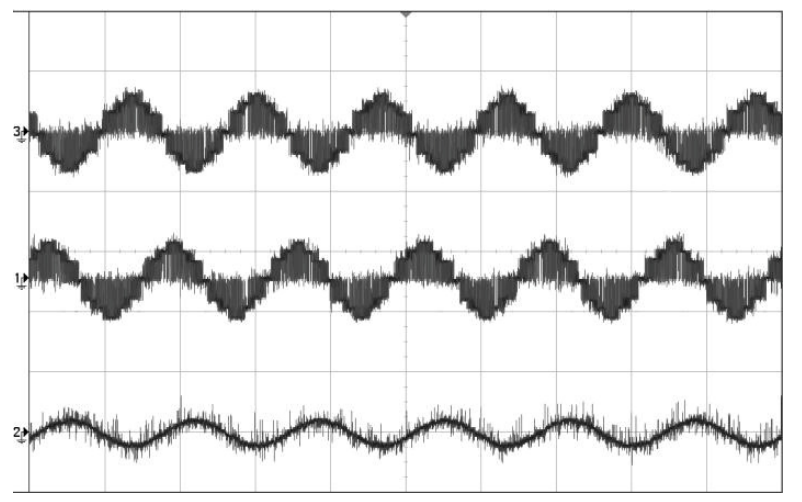

Fig. 7. Resultados experimentais: tensões (200V/div) e corrente $(6 \mathrm{~A} / \mathrm{div})$ de fase da carga trifásica.

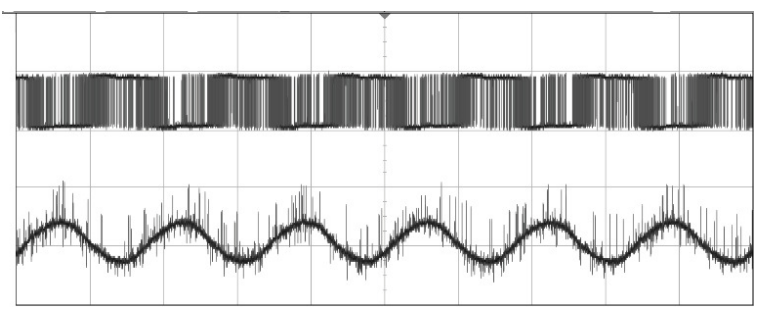

(a)

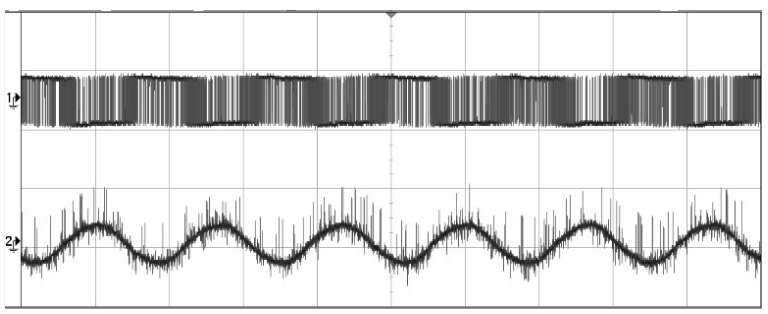

(b)

Fig. 8. Resultados experimentais: (a) tensão de pólo - $v_{10}(50 \mathrm{~V} / \mathrm{div})$ e corrente no secundário do transformador ( $3 \mathrm{~A} /$ div); (b) tensão de pólo - $v_{a 0}(50 \mathrm{~V} / \mathrm{div})$ e corrente no primário do transformador (3A/div). 


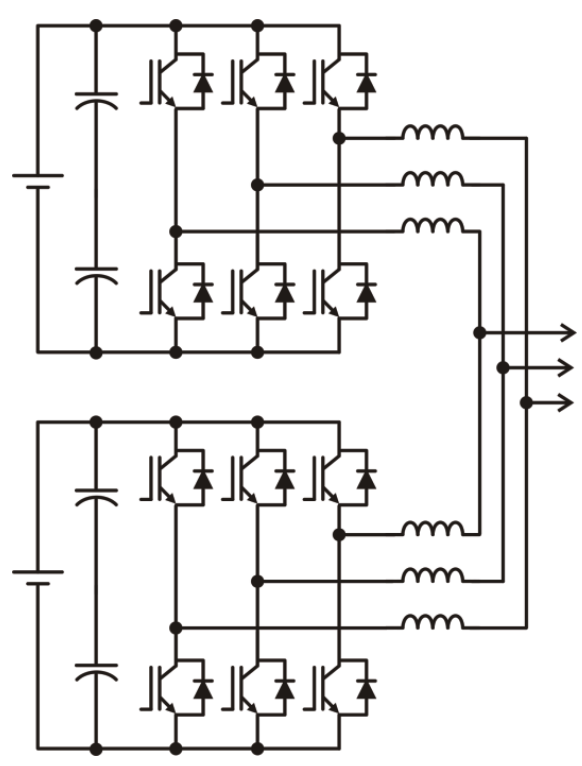

Fig. 9. Inversor em paralelo.

\section{COMPARAÇÃO ENTRE O INVERSOR ATS E INVERSORES CONVENCIONAIS}

Nesta seção será apresentada uma comparação entre o inversor proposto (Inversor ATS) e duas configurações de conversores estáticos de potência. Uma destas configurações é mostrada na Figura 9 (inversor em paralelo), enquanto a outra topologia empregada na comparação é o inversor trifásico convencional de três braços. O conversor paralelo foi escolhido porque possui os mesmo número de braços e elementos magnéticos em sua saída, como observado no inversor ATS.

$\mathrm{Na}$ análise comparativa, alguns aspectos serão considerados, tais como: i) distorção harmônica das tensões geradas pelos inversores, ii) tensão do barramento capacitivo e iii) custos das configurações.

\section{A. Distorção Harmônica}

A THD da tensão de fase da carga trifásica é calculada considerando a seguinte expressão

$$
T H D(p)=\frac{1}{a_{1}} \sqrt{\sum_{i=2}^{p}\left(a_{i}\right)^{2}}
$$

Onde $a_{1}$ é a amplitude da tensão fundamental, $a_{i}$ é a amplitude da harmônica de ordem $i$ e $p$ é o número de harmônicas levadas em consideração.

Mostra-se na Figura 10 uma curva da THD da tensão de fase da carga em função do índice de modulação, considerando as três topologias de conversores estáticos sob comparação, ou seja, Inversor ATS (Figura 1), inversor em paralelo [Figura 9] e inversor de três braços convencional.

\section{B. Tensão do Barramento Capacitivo}

Mostra-se na Tabela I a tensão do barramento exigida para cada uma das configurações consideradas na comparação. Nesta tabela, a tensão do barramento está normalizada com relação à tensão de fase da carga trifásica, que é de $1 p u$. Na Tabela I $\Delta$ é a queda de tensão nas indutâncias do inversor em paralelo.

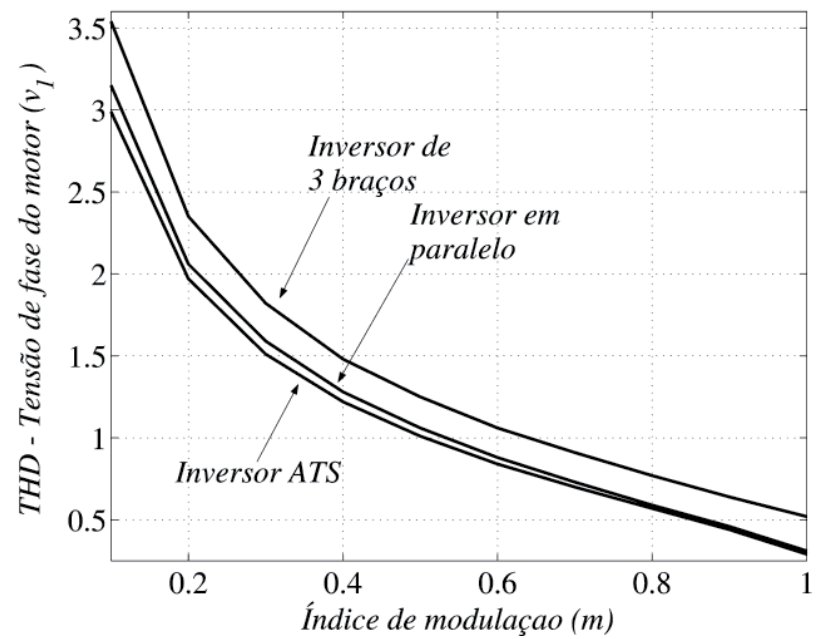

Fig. 10. THD da tensão de fase da carga trifásica em função do índice de modulação.

TABELA I

Tensão do barramento capacitivo

\begin{tabular}{ccc}
\hline $\begin{array}{c}\text { Inversor } \\
\text { ATS }\end{array}$ & $\begin{array}{c}\text { Inversor de três } \\
\text { braços }\end{array}$ & $\begin{array}{c}\text { Inversor em } \\
\text { paralelo }\end{array}$ \\
\hline \multirow{2}{*}{$0.86 p u$} & $\sqrt{3} p u$ & $(\sqrt{3}+\Delta) p u$ \\
& & \\
\hline
\end{tabular}

$\mathrm{O}$ inversor de três braços convencional necessita de uma tensão de barramento igual a $\sqrt{3} p u$ que é a tensão de linha exigida pela carga, como o conversor ATS necessita de apenas metade da tensão do conversor de três braços, sua tensão do barramento é igual a $0.86 p u$.

A partir desta comparação, percebe-se o benefício trazido pelo Inversor ATS no que diz respeito à tensão do barramento capacitivo necessária para que este inversor possa gerar 1pu de tensão na fase da carga de tensão na fase. Isto é conseguido graças à ação de tensão série do inversor proposto.

\section{Custos das Configurações}

Como observado anteriormente, para que as configurações proposta e paralela gerem uma tensão de saída com mesma amplitude, é necessário aumentar a tensão na entrada do inversor paralelo. Em contrapartida, sabe-se que a corrente processada pelo conversor paralelo é menor que aquela observada no Inversor ATS. Logo, o investimento inicial do Inversor ATS poderá ser maior que o investimento do inversor em paralelo se as chaves que processam uma corrente de amplitude maior forem mais caras que aquelas que processam uma tensão maior. Contudo, se as chaves com maior tensão forem mais caras que as de maior corrente, isso levaria a um aumento no preço da configuração paralelo, tornando assim o Inversor ATS mais competitivo. Adicionalmente, a presença de dispositivos magnéticos na saída dos inversores, bem como a relação dos preços destes 
dispositivos com o preço das chaves de potência, irá influenciar o preço final de cada topologia.

Tanto o inversor ATS quanto o inversor em paralelo possuem investimentos iniciais maiores que o inversor de três braços. No entanto, a escolha do Inversor ATS é justificada, se a distorção harmônica gerada pelo inversor e o melhor aproveitamento da tensão do barramento são fatores críticos em uma dada aplicação.

\section{Perdas por Chave}

A estimação de perdas foi realizada através de um modelo de regressão, o qual foi obtido a partir de um procedimento experimental, como apresentado em [23]. A chave de potência usada foi um módulo dual IGBT CM50DY-24H (POWEREX) acionada por SKHI-10 (SEMIKRON). O modelo das perdas da chave inclui: a) perdas de condução no IGBT e no diodo, b) perdas de disparo no IGBT, c) perdas de bloqueio no IGTB, e d) perdas de recuperação reversa no diodo.

Para garantir uma comparação de perdas justa, as condições de operação dos inversores convencional (com três braços) e proposto (Inversor ATS) foram mudadas. Assim, o critério utilizado foi que ambos inversores garantissem a mesma amplitude de tensão na carga com a mesma distorção harmônica. A tensão do elo cc foi $400 \mathrm{~V}$ para o inversor convencional e $200 \mathrm{~V}$ para o conversor proposto. E a freqüência de chaveamento foi de $10 \mathrm{kHz}$ e $8 \mathrm{kHz}$ para o convencional e proposto, respectivamente.

Uma importante característica para inversores fonte de tensão foi considerada, ou seja, perdas por chave que fornece um importante referencial a respeito do stress das chaves de potência para um dado inversor. A partir deste indicativo é possível estimar uma possível falha no inversor [24]. Mostrase na Figura 11 as perdas por chave no inversor convencional e proposto. Como esperado, as perdas de condução por chave é praticamente a mesma para os dois inversores, já que as chaves de cada inversor processam as mesmas correntes (corrente da carga). Por outro lado, as perdas de comutação por chave são consideravelmente menores no Inversor ATS, já que a tensão e freqüência de chaveamento são reduzidas. As perdas totais por chave mostradas na mesma figura referem-se à soma das parcelas de condução e chaveamento em cada chave. Note que, as perdas totais por chave na Figura 11 para o inversor ATS têm valor de aproximadamente metade daquele observado para $o$ conversor de três braços, o que implicará, considerando as topologias completas, em praticamente as mesmas perdas totais para as duas topologias, ou seja, praticamente o mesmo rendimento.

As perdas por chave no inversor em paralelo não foram mostradas neste gráfico, mas são ainda ligeiramente menores que a do Inversor ATS, isso se deve ao fato de a corrente processada ser metade daquela processada por cada chave do Inversor ATS.

\section{CONCLUSÕES}

Neste artigo foi proposto um inversor nomeado de Inversor ATS devido à ação de tensão em série que caracteriza o circuito. Foi apresentada uma análise teórica do

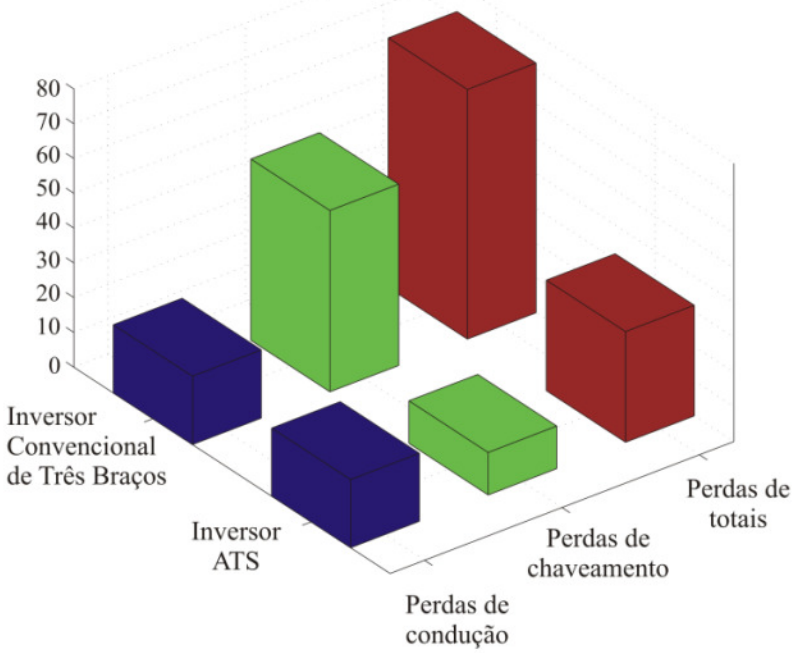

Fig. 11. Comparação de perdas por chave no inversor convencional de três braços e Inversor ATS.

conversor proposto, mostrando sua operação, a estratégia de controle PWM, resultados de simulação e experimentais, bem como foi fornecida uma comparação com dois inversores conhecidos na literatura técnica.

Os benefícios do Inversor ATS estão relacionadas com a ação de tensão série obtidas neste circuito, o que proporciona melhor aproveitamento da tensão do barramento e baixa distorção harmônica na tensão aplicada à carga.

\section{REFERENCIAS}

[1] J. Rodriguez, J.-S. Lai, and F. Z. Peng, "Multilevel inverters: A survey of topologies, controls, and applications," IEEE Trans. Ind. Electron., vol. 49, pp. 724-738, Aug. 2002.

[2] J. I. Leon, S. Vazquez, R. Portillo, L. G. Franquelo, J. M. Carrasco, P. W., Wheeler, and A. J. Watson, "Threedimensional feedforward space vector modulation applied to multilevel diode-clamped converters," IEEE Trans. Ind. Electron., vol. 56, pp. 101-109, Jan. 2009.

[3] M. Saeedifard, R. Iravani, and J. Pou, "A space vector modulation strategy for a back-to-back five-level hvdc converter system," IEEE Trans. Ind. Electron., vol. 56, pp. 452.466, Feb. 2009.

[4] S. Busquets-Monge, S. Alepuz, J. Rocabert, and J. Bordonau, "Pulse width modulations for the comprehensive capacitor voltage balance of n-level three-leg diode-clamped converters," IEEE Trans. Power Electron., vol. 24, pp. 1364-1375, May 2009.

[5] G. J. Su, "Multilevel dc-link inverter," IEEE Trans. Ind. Appl., vol. 41, pp. 848-854, May/June 2005.

[6] F. H. Khan, L. M. Tolbert, and W. E. Webb, "Start-up and dynamic modeling of the multilevel modular capacitor-clamped converter," IEEE Trans. Power Electron., vol. 25, pp. 519-531, Feb. 2010.

[7] M. L. Heldwein, S. A. Mussa, and I. Barbi, "Three-phase multilevel pwm rectifiers based on conventional bidirectional converters," IEEE Trans. Power Electron., vol. 25, pp. 545-549, March 2010. 
[8] P. Hammond, "A new approach to enhance power quality for medium voltage ac drives," IEEE Trans. Ind. Appl., vol. 33, p. 202-208, Jan./Feb. 1997.

[9] L. Shuai and K. A. Corzine, "Advanced control and analysis of cascaded multilevel converters based on $\mathrm{p}-\mathrm{q}$ compensation," IEEE Trans. Power Electron., vol. 22, pp. 1242-1252, July 2007.

[10] J. Wen and K. M. Smedley, "Hexagram inverter for medium-voltage six-phase variable-speed drives," IEEE Trans. Ind. Electron., vol. 55, pp. 2473-2481, June 2008.

[11] R. Gupta, A. Ghosh, and A. Joshi, "Switching characterization of cascaded multilevel-invertercontrolled systems," IEEE Trans. Ind. Electron., vol. 55, pp. 1047-1058, March 2008.

[12] M. Malinowski, K. Gopakumar, J. Rodriguez, and M. Perez, "A survey on cascaded multilevel inverters," IEEE Trans. Ind. Electron., vol. 1, pp. 1-10, Dec. 2009.

[13] D. G. Holmes and B. P. McGrath, "Opportunities for harmonic cancellation with carrier-based pwm for a twolevel and multilevel cascaded inverters," IEEE Trans. Ind. Appl., vol. 37, pp. 574-582, March-April 2001.

[14] J. Rodriguez, L. Moran, J. Pontt, P. Correa, and C. Silva, "A high-performance vector control of an 11-level inverter," IEEE Trans. Ind. Electron., vol. 50, pp. 80-85, Feb. 2003.

[15] D. Zhong, L. M. Tolbert, B. Ozpineci, and J. N. Chiasson, "Fundamental frequency switching strategies of a seven-level hybrid cascaded h-bridge multilevel inverter," IEEE Trans. Power Electron., vol. 24, pp. 2533, Jan. 2009

[16] P. Barriuso, J. Dixon, P. Flores, and L. Moran, "Faulttolerant reconfiguration system for asymmetric multilevel converters using bidirectional power switches," IEEE Trans. Ind. Electron., vol. 56, pp. 13001306, April 2009.

[17] S. G. Song, F. S. Kang, and S. J. Park, "Cascaded multilevel inverter employing three-phase transformers and single dc input," IEEE Trans. Ind. Electron., vol. 56, pp. 2005-2014, June 2009.

[18] R. M. Cuzner, D. J. Nowak, A. Bendre, G. Oriti, and A. L. Julian, "Mitigating circulating common-mode currents between parallel soft-switched drive systems," IEEE Trans. Ind. Appl., vol. 43, pp. 1284-1294, Sept./Oct. 2007.

[19] C. B. Jacobina, E. C. dos Santos Jr., E. R. C. da Silva, M. B. R. Correa, A. M. N. Lima, and T. M. Oliveira, "Reduced switch count multiple three-phase ac machine drive systems," IEEE Trans. Power Electron., vol. 23, pp. 966-976, Mar. 2008.

[20] SEMIKRON Products. Available at http://www.semikron.com. 2010.

[21] C. B. Jacobina, A. M. N. Lima, E. R. C. da Silva, R. N. C. Alves, and P. F. Seixas, "Digital scalar pulse-width modulation: a simple approach to introduce nonsinusoidal modulating waveforms," IEEE Trans. Power Electron., vol. 16, pp. 351-359, May 2001.

[22] V. Blasko, "Analysis of a hybrid PWM based on modified space-vector and triangle-comparison methods," IEEE Trans. Ind. Appl., vol. 33, pp. 756-764, May/June 1996.
[23] M. C. Cavalcanti, E. da Silva, D. Boroyevich, W. Dong, and C. B. Jacobina, "A feasible loss model for IGBT in soft-switching inverters," in Proc. IEEE PESC, pp. 1845.1850, 2003.

[24] M. Aten, G. Towers, C. Whitley, P. Wheeler, J. Clare, and K. Bradley, "Reliability comparison of matrix and other converter topologies". Aerospace and Electronic Systems, IEEE Transactions on, vol. 42, pp. 867.875 , July 2006.

[25] E. Cengelci, S. U. Sulistijo. B. O. Woo, P. Enjeti, R. Teodorescu and F. Blaabjerg, "A New Medium-Voltage PWM Inverter Topology for Adjustable-Speed Drives", Industry Applications, IEEE Transactions on, vol. 35, pp. 628-637, May-June, 1999.

[26] J. Salmon, J. Ewanchuk, and A. Knight, "Pwm inverters using split-wound coupled inductors", Industry Applications, IEEE Transactions on, vol. 45, pp. 2001 .2009, Nov.-Dec. 2009.

[27] F. B. Grigoletto, H. Pinheiro, "Modulação space vector estendida a conversores estáticos com braços em paralelo", Eletrônica de Potência - SOBRAEP, vol. 16, no. 1, pp. 56-67, Dez 2010/fev. 2011.

[28] F. B. Grigoletto, H. Pinheiro, "Método de Modulação PWM para Equilíbrio das Tensões dos Capacitores do Barramento $\mathrm{CC}$ em Conversores Multiníveis com Diodos de Grampeamento", Eletrônica de Potência SOBRAEP, vol. 14, no. 2, pp. 63-74, Maio 2009.

[29] T. R. Fernandes Neto, R. S. T. Pontes, "Design of an Elevator Prototype Propelled by a Linear Induction Motor", Eletrônica de Potência - SOBRAEP, vol. 14, no. 3, pp. 181-187, Agosto 2009.

[30] F. K. de A. Lima, E. H. Watanabe, P. Rodriguez, A. Luna, "Controle de Gerador de Indução Duplamente Alimentado Diante de Afundamento de Tensão", Eletrônica de Potência - SOBRAEP, vol. 14, no. 3, pp. 189-199, Agosto 2009.

[31] V. P. Pinto, J. C. T. Campos, N. Rocha, C. B. Jacobina, "Controlador Robusto Multivariável com Ação Integral Aplicado em um Sistema de Geração Eólica", Eletrônica de Potência - SOBRAEP, vol. 16, no. 2, pp. 147-157, Agosto 2009.

[32] A. V. Rocha, G. J. França, M. E. dos Santos, H. de Paula, B. J. Cardoso Fl., "A Fault-Resilient Implementation of Three-Level NPC IGCT-Based Converters", Eletrônica de Potência - SOBRAEP, vol. 16, no. 2, pp. 168-176, Agosto 2009.

\section{DADOS BIOGRÁFICOS}

Euzeli Cipriano dos Santos Junior obteve diploma de engenheiro e de Mestre em Engenharia Elétrica da Universidade Federal de Campina Grande, em 2004 e 2005, respectivamente, e o Diploma de Doutor em Engenharia Elétrica em 2007 pela Universidade Federal de Campina Grande, participou de doutorado-sanduíche na Texas A\&M University, entre 2006 e 2007. De 2006 a 2009 foi professor do Centro Federal de Educação Tecnológica da Paraíba. De Dezembro de 2010 a Março de 2011 foi professor visitante na Universidade de Siegen, Alemanha. Desde 2009 é professor da UFCG. Atualmente é conselheiro do Ramo Estudantil do IEEE - UFCG. 